\title{
Research of Interior Decoration System Based on VRML
}

\author{
Yang Kuang ${ }^{1, a}$, Jie Jiang ${ }^{2, b}$ \\ ${ }^{1}$ Institute of Education, Jiang Xi Science and Technology Normal University, NanChang, China \\ ${ }^{2}$ Institute of Education, Jiang Xi Science and Technology Normal University, NanChang, China \\ aemail: ky_super@163.com, bemail: janyjiang@163.com
}

Keywords: VRML; Interior Decoration; Virtual Reality

\begin{abstract}
In the process of interior decoration, customers don't know which is more suitable. This paper introduced how to realize the virtual interior decoration effect through the VRML. Customers can compare the different kinds of effect drawing, they can see the end of the decoration effect, so it is convenient for users to choose the decoration scheme last. This system is a kind of desktop Virtual reality system. Users can compare interior decoration wallpaper, furniture and other decorative schemes of different floor through the cursor operation.
\end{abstract}

\section{Introduction}

Virtual reality technology allows users to experience in a virtual 3D world, and provide a visual, hearing and other direct and natural real-time perception. The common Virtual reality system are, desktop virtual reality system, immersive virtual reality system, augmented reality systems. The researchers believe that, these three kinds of system have no significant difference in Kansei Evaluation of products. Desktop virtual reality system can not only meet the requirements, but also the cost is low[1]. VRML is a common and very practical technology of desktop virtual reality system. VRML provides about 54 types of nodes are used in construct the scene modeling, there are 6 kinds of interpolation nodes create animation effects, and there are 7 kinds of sensor nodes perform sensing and effect. It also can be embedded JAVA procedures to achieve human-computer interaction, so as to greatly expand its liquidity.

\section{System Function}

In this system, the user download the VRML 3D scene file that the suffix is WRL request to the server through the browser model, then display the 3D scene through VRML. JavaScript access the file through the application program interface, so as to realize the user interaction with the 3D scene. The server provide users the documents or other information by monitoring the 3D events. As shown in figure 1.

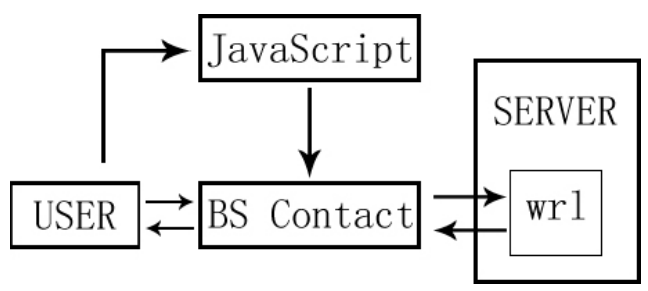

Figure 1. The function of system

The function of system is, provide indoor scene space such as living room, restaurant model, the user choice decoration, move furniture and the help. There is no need designers have professional design or computer literacy, which can make the effect of demand of interior decoration according to their idea. Visualization of the mode of operation has overcome the disadvantages of traditional design method, immersion and interaction characteristics of virtual reality technology brings convenience for users, improve effectively the success rate of design. The system includes the following several specific functions:

Indoor scene roaming function. The user can feel in the scene before and after the replacement of virtual decoration. 
The scene effect transform. Achieved the scene transform through the menu. Such as: wallpaper, furniture and other transformation.

Menu functions. The realization of two-dimensional menu function control virtual decoration in 3D scene.

\section{Construct the Scene Model}

The build quality is closely related to the availability of the whole system VRML scene model, if there is no real fine scene model, the software cannot attract users.

The visualization software tool 3DS through MAX modeling, the model can directly guide to WRL format file, and use the 3DS MAX more suitable for production of complex and irregular model, greatly improving production efficiency. The model should pay attention to the following points:

a. The model satisfies the basic requirement of the show will be simplified as much as possible, the model is too complex will bring too much burden on the system, reduce the scene running speed, is not conducive to use online.

b. Deleted invisible surface in scene, such as: the bottom of the object can be removed.

c. For repeated occurrences of objects can be expressed with bulletin boards, greatly reduce model complexity.

d. Use real pictures model complex object can simulate the complex effect.

e. Note that the 3DS MAX scene unit and VRML unit in the VRML scene is different, a unit is a unit, whether in the 3DS MAX is $1 \mathrm{~m}$ or $1 \mathrm{~cm}$.

When modeling is completed, we give material texture to the model through the relevant pictures, or direct acquisition of object texture, The picture as much as possible with a high resolution camera field acquisition, the texture detail is perfect as possible. After the completion of the shooting, also need to cut through image processing software, collage, get the final texture palette.

There is no light in the VRML concept. Show the effects of light and shadow will be on the completed model for baking, the surface generated scene lighting effects directly generated to model textures, baked mapping as shown in figure 2. Finally, adjust the texture coordinates to obtain the scene effect.

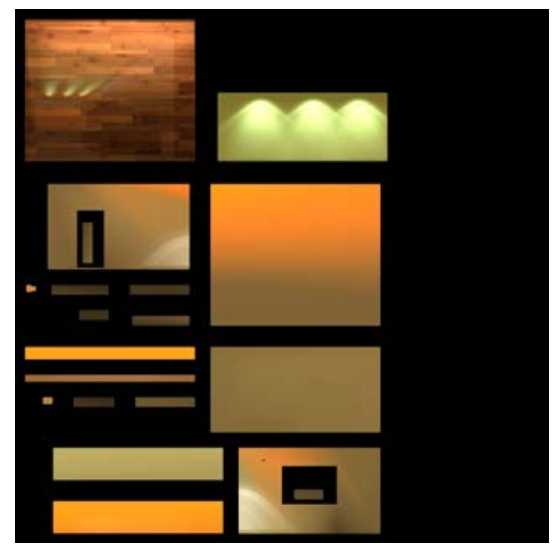

Figure 2. The scene effect

\section{Interaction Principle}

The scene of the model formed by the nodes, node formed by the domain and event. Domain definition node attributes, the interaction between the user and the scene of the event is defined, the virtual world is dynamic. Create a route between nodes, A node control another node by sending an event. This method can realize the simple interaction, in order to realize the complex interactive, we must use an external application. A method for calling external programs is to use the Script [2] node, it similar to other standard node, only a little different, a Script node is expanded by the user, reached the field event will automatically move to the related procedures in the Script node. It can pass the TouchSensor and TimeSensor sensor and the JavaScript program directly to complete the 
parameter on the node change inside the VRML, We need to trigger interaction. Users schedule the initial state, node parameters is first initialized work.

\section{Construct a Navigation Mmenu}

Using the prototype (PROTO) to create a new node [3] in the VRML documentation, if defined as the prototype, we can use the VRML standard node type to use the prototype, menu and two-dimensional elements can be used to construct. Navigation menus can be defined:

EXTERNPROTOMenu[

exposedField SFVec2f size

exposedField MFString img

fieldMFString state \#up or down

exposedField MFNode buttons

exposedField SFVec2f buttonsize

]

It can also achieve the menu through the definition of a Transform node, which constructs a menu_floor with no thickness rectangular, and then paste the picture menu information in a rectangular, define a TouchSensor node in response to a user's click operation. Menu _ floor nodes defined code as follows:

DEF menu_floor Transform \{

rotation $-1.03100-1.056$

children [DEF floor TouchSensor \{\}

Shape $\{\ldots\}$

texture ImageTexture

\{url "maps/floor.jpg"\} \}

... $\}\}]\}$

The user can change other state triggering event or start a VRML animation accord needing in the scene, as shown in Figure 3. Opens the menu through the browser window can be on the scene in the floor texture replacement. Replace the floor before and after comparison as shown in Figure 4. Change from the reflective material into sub light material, the decorative effect is obvious, and the user can roam in the room, feel the floor material changes, to bring about changes in the overall effect of the room.

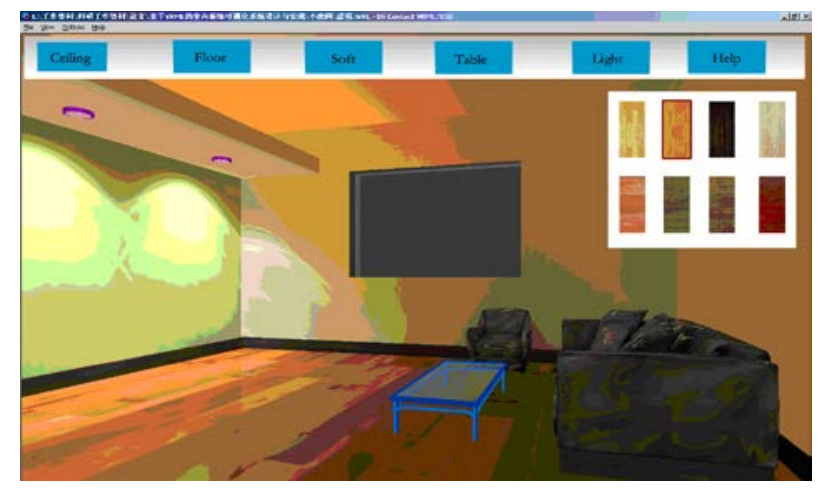

Figure 3. Change floor material

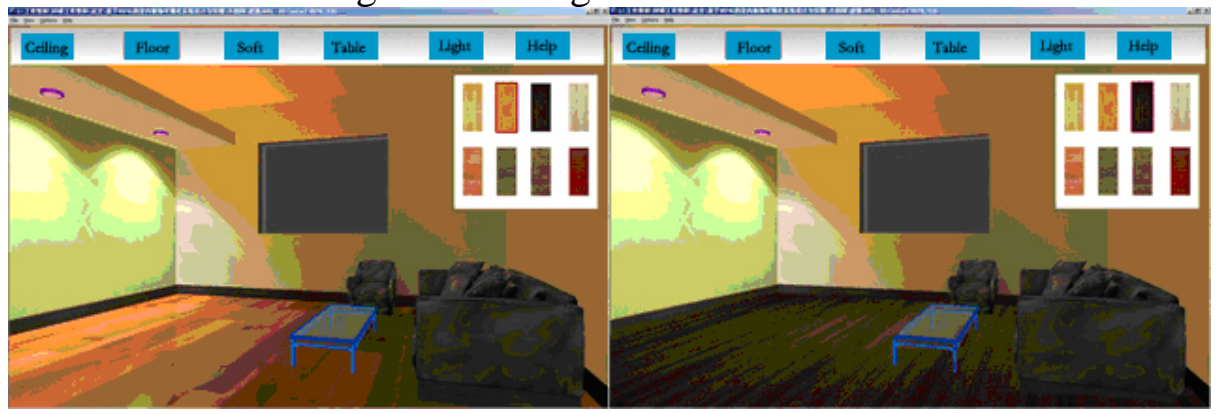

Figure 4. The effect of room 
Look below the implementation process, the user click the navigation bar on the floor button, scene joint main menu via route ROUTE floor button event to the floor dialog into the event, open floor material dialog box, the code is as follows

ROUTE button_floor.isActive to dialog_floor.openit

The system set button events dialog and dialog and program design through the Script node. As to the floor dialog box settings into the event: eventIn sfbool button_touched. On the floor of a button in a dialog box is set in the event: eventIn sftime flrbutton0_path0_clicked, set the event: eventOut mfstring flrtextrue_changed0. And the programming of these events, such as the pair and flrbutton_path0_clicked code is as follows:

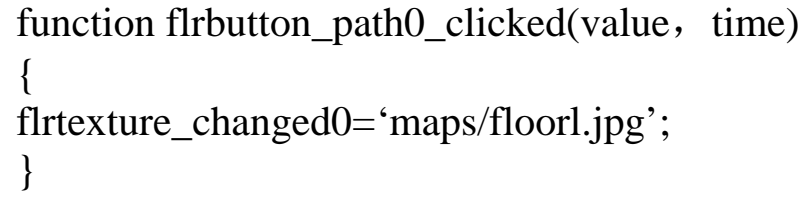

When the user selects a floor material on the floor dialog_floor dialog box, the system through the ROUTE connection and flrbutton_path0 routing the event touchTime to the Script node into the event flrbutton_Path0_clicked, then connect the Script node of the event and the flrtexture_changed1 to the URL domain image texture node ImageTexture plate, so are transformed to the floor material. The relevant code as follows:

ROUTE flrbutton_path0.touchTime to scr.flrbutton_path0_clicked

ROUTE scr.flrtexture_changed0 to floor.url

\section{Conclusion}

Interior decoration of visualization design system based on VRML, can allow the user freely design based on their living environment, help users experience the true final effect of their own design, without the need to have the professional knowledge of computer or design. The system use a desktop virtual reality, without the premise of hardware equipment is explored on the interior visualization, virtual reality software and real environment combined, process to the user experience design of interior decoration. Represented by the VRML desktop virtual reality development pattern is characterized by low cost, good effect, suitable for individuals and small team of developers.

\section{References}

[1] LEE S, CHEN T, KIM J, et al. Affective Property Evaluation of Virtual Product Designs[C]. Proceedings - Virtual Reality Annual International Symposium, Chicago, IL, 2004.

[2] Ande Jiang, VRML from entry to the master, BeiJing, Academic Press,2002

[3] Qian Xing Wang. VRML virtual modeling combat exercises. BeiJing, China water conservancy and Hydropower Press, 2001 